\title{
Surface enhanced infrared absorption spectroscopy based on gold nanostars and spherical nanoparticles
}

\author{
Olga Bibikova a, b, c, d, Julian Haas ${ }^{\text {b }}$, Ángela I. López-Lorente ${ }^{\mathrm{b}}$, Alexey Popov ${ }^{\mathrm{a}, \mathrm{e}, \mathrm{f}}$,

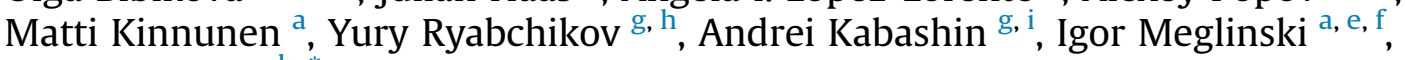 \\ Boris Mizaikoff ${ }^{b, *}$
}

a Optoelectronics and Measurement Techniques Research Unit, University of Oulu, 90014 Oulu, Finland

${ }^{\mathrm{b}}$ Institute of Analytical and Bioanalytical Chemistry, Ulm University, 89081 Ulm, Germany

${ }^{c}$ Art Photonics GmbH, 12489 Berlin, Germany

${ }^{\mathrm{d}}$ Research-Educational Institute of Optics and Biophotonics, Saratov National Research State University, 410012 Saratov, Russia

e ITMO University, 197101 St Petersburg, Russia

${ }^{\mathrm{f}}$ Interdisciplinary Laboratory of Biophotonics, Tomsk National Research State University, 634050 Tomsk, Russia

${ }^{g}$ Aix-Marseille University, CNRS, UMR 7341 CNRS, LP3, Campus de Luminy, Case 917, F-13288 Marseille Cedex 9, France

${ }^{\mathrm{h}}$ P.N. Lebedev Physical Institute of Russian Academy of Sciences, 199991 Moscow, Russia

${ }^{i}$ National Research Nuclear University “MEPhI”, Institute of Engineering Physics for Biomedicine (PhysBio), Bio-Nanophotonics Lab., 115409 Moscow, Russia

\section{H I G H L I G H T S}

- Signal enhancement from two analytes at plasmonic nanostars and nanospheres were compared for SEIRAS in IR-ATR.

- The signal obtained from nanostars was at least 2-times higher in comparison with nanospheres.

- Up to 10-times signal enhancement at plasmonic nanostars for SEIRAS was observed.

- Dependence of enhancement on the number of nanostars at the internal reflection element surface was demonstrated.

- SEIRA signal was correlated to the concentration of analyte molecules present within the evanescent field.

\section{A R T I C L E I N F O}

\section{Article history:}

Received 23 March 2017

Received in revised form

24 July 2017

Accepted 24 July 2017

Available online $\mathrm{xxx}$
G R A P H I C A L A B S T R A C T

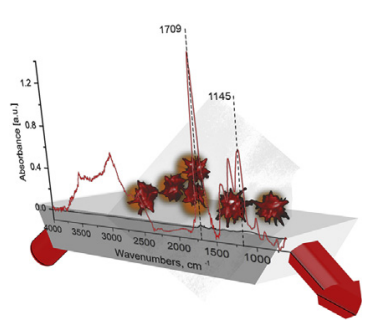

Abbreviations: ATR, attenuated total reflection; AuNSts, gold nanostars; BSA, bovine serum albumin; c-NPs, citrate-capped spherical nanoparticles; CTAB, cetyl-

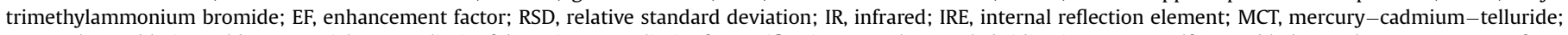

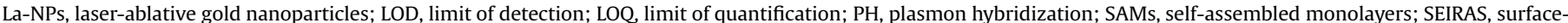

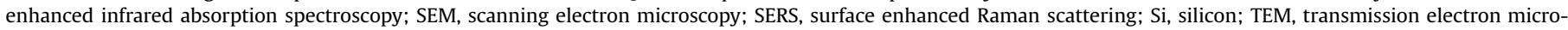
scopy; TGA, thioglycolic acid.

* Corresponding author.

E-mail address: boris.mizaikoff@uni-ulm.de (B. Mizaikoff). 
Keywords:

FTIR spectroscopy

SEIRAS

Plasmonic

Gold nanostars

Gold nanospheres enhancement of signals for bovine serum albumin (BSA) protein on plasmonic nanostructure-based films deposited on a silicon (Si) internal reflection element (IRE) compared to bare Si IRE. The dependence of the observed enhancement on the amount of AuNSts present at the surface of the IRE has been demonstrated. Quantitative studies with both, TGA and BSA were performed, observing that the SEIRA signal can be correlated to the concentration of analyte molecules present within the evanescent field. The calibration curves in the presence of the AuNSts showed enhanced sensitivity as compared with the bare Si IRE. We finally compare efficiencies of anisotropic AuNSts and spherical citrate-capped and "bare" laser-synthesized gold nanoparticles as SEIRAS substrates for the detection of TGA and BSA. The signal obtained from AuNSts was at least 2 times higher for TGA molecules in comparison with spherical gold nanoparticles, which was explained by a more efficient generation of hot spots on anisotropic surface due to the presence of sharp edges, tips or vertices, leading to a high electric field strength surrounding the AuNSts.

() 2017 Elsevier B.V. All rights reserved.

\section{Introduction}

The enhanced electromagnetic field strength of gold nanoparticles (AuNPs) due to plasmon excitation [1] may be exploited for surface-enhanced vibrational spectroscopies such as surfaceenhanced infrared absorption spectroscopy (SEIRAS) [2]. SEIRAS is capable of providing molecular information on analyte molecules located in close vicinity to metallic islands or nanostructures. Furthermore, material-specific vibrational absorptions in the IRfingerprint region enable unambiguous molecular identification [3]. SEIRA in an attenuated total reflection (ATR) configuration was first reported by Hartstein et al. [2]. ATR spectroscopy takes advantage of total internal reflections within an internal reflection element (IRE) or waveguide [4,5]. SEIRAS in an ATR configuration was previously used to investigate chemical interactions between SEIRA-active layers and various chemicals [6], for the analysis of biological species [7], to monitor the adsorption kinetics of bovine hemoglobin on AuNP films with an improved detection sensitivity (i.e., up to 3 orders of magnitude) [8] and to study the adsorption behavior of cytochrome $C$ at carboxylic acid and hydroxyl terminated self-assembled monolayers immobilized at gold surfaces [9]. In addition, López-Lorente et al. in situ monitored the synthesis of bare AuNPs mediated by stainless steel as reducing agent via SEIRAATR spectroscopy [10,11].

Typically, as a first step in SEIRAS studies AuNPs [8,12,13] are periodically structured at the crystal surface providing for twodimensional arrays. Deposition techniques include sputtering [14], lithography [15] and vapor phase or wet chemical deposition [16]. In a second step, analyte molecules are administered at the arrayed gold surface. In the present study, gold nanostructures were interacted and assembled with analyte molecules in colloidal solution prior to the formation of AuNP arrays at Si ATR waveguide surfaces by adapting a procedure from Seelenbinder et al. [17].

Notably, the signal enhancement strongly depends on the sharpness of AuNP edges, tips or vertices, which are directly related to the intensity of the electromagnetic field surrounding AuNPs [18]. From that point of view, anisotropic AuNPs such as nanorods [19], nanoshells [20], or nanocages [21] are potential amplifiers. However, these types of AuNPs have several limitations including elaborate and time-consuming synthesis. In contrast, AuNSts synthesized via seed-mediated methods [22] require a minimum of neat compounds in a straightforward procedure, and appear to be excellent electromagnetic field enhancers owing to the large number of hot spots at the surface associated with the sharp tips. Moreover, according to the plasmon hybridization (PH) model [23] the core-tip structure serves as an antenna increasing both, the excitation cross section and the electric field enhancement of nanostar plasmon modes [24]. To the best of our knowledge, only a few research groups have applied IR-ATR spectroscopy for monitoring the growth kinetics of such particles [25] and have analyzed the surface chemistry of the AuNSts in details [26]. Last but not least, AuNSts have not been applied in ATR-SEIRAS to date.

In this work, different gold nanostructures (nanostars, ligandprotected or bare spherical nanoparticles) have been deposited together with analytes on a Si IRE and their responses in ATR SEIRAS compared. In our tests, so prepared SEIRAS substrates were used to study the signal enhancement of bovine serum albumin protein (BSA) and thioglycolic acid (TGA). TGA, which was previously investigated in various analytical studies [27,28], was selected as an exemplar of small molecule. BSA, was selected as an extensively studied example of a large molecule within the family of serum albumins, and is among the most important blood carrier proteins [29]. The present study demonstrates that for both TGA and BSA examples an enhancement of the IR-signal due to the presence of the AuNPs was achieved, as compared to IR-ATR results obtained at the bare Si surfaces. This enhancement directly related to the amount of Au nanostructures present within the evanescent field. SEIRA signals obtained with AuNSts have also been compared to results obtained at alternative gold nanostructures with a spherical shape using both, citrate-capped and bare Au nanospheres. The presented results demonstrate that the signal enhancement produced by AuNSts is elevated in comparison to spherical AuNPs, which is explained by a much larger electromagnetic field strength at sharp tips on their surface. These data demonstrate a high potential of gold nanostars in surface-enhanced infrared absorption spectroscopy.

\section{Materials and methods}

\subsection{Chemicals}

Gold (III) chloride trihydrate $\left(\mathrm{HAuCl}_{4} \times 3 \mathrm{H}_{2} \mathrm{O}\right)$, trisodium citrate dihydrate $\left(\mathrm{C}_{6} \mathrm{H}_{5} \mathrm{O}_{7} \mathrm{Na}_{3} \times 2 \mathrm{H}_{2} \mathrm{O}\right)$, hydrochloric acid $(\mathrm{HCl}), \mathrm{L}(+)-$ ascorbic acid (AA), silver nitrate $\left(\mathrm{AgNO}_{3}\right)$, thioglycolic acid (TGA), bovine serum albumin (BSA) were purchased from Sigma-Aldrich (St. Louis, USA) at the highest purity grade available. Milli-Q water (resistivity $18.2 \mathrm{M} \Omega \mathrm{cm}$ at $25^{\circ} \mathrm{C}$; Millipore, USA) was used in all preparations. Prior to synthesis, the glassware was washed with a mixture of nitric acid and hydrochloric acid $\left(\mathrm{HNO}_{3} / \mathrm{HCl} 1: 3\right.$ mixture, aqua regia).

\subsection{Synthesis of gold nanostars}

AuNSts were fabricated by a modified seed-mediated growth protocol by Yuan et al. [29] with minor modifications [30]. Seeds were prepared using the citrate reduction method introduced by 
Grabar [31]. For the AuNSt synthesis, $150 \mu \mathrm{L}$ of $1 \mathrm{M} \mathrm{HCl}$ and $1.5 \mathrm{~mL}$ of the seed solution were added to $50 \mathrm{~mL}$ of $0.25 \mathrm{mM} \mathrm{HAuCl}_{4}$ water solution at room temperature under vigorous stirring. Then, $1.5 \mathrm{~mL}$ of $2 \mathrm{mM} \mathrm{AgNO}_{3}$ and $0.75 \mathrm{~mL}$ of $0.1 \mathrm{M} \mathrm{AA}$ were simultaneously added. The solution was stirred for $30 \mathrm{~s}$ while its color turned from light red to dark grey. The AuNSts were concentrated by centrifugation to an Au amount equal to $500 \mu \mathrm{g} \mathrm{mL}^{-1}$, as determined via Inductively coupled plasma atomic emission spectroscopy (ICPAES).

\subsection{Synthesis of spherical nanoparticles}

\subsubsection{Citrate-capped spherical nanoparticles (c-NPs)}

40-nm citrate-capped spherical nanoparticles (c-NPs) were prepared by the reduction of $\mathrm{HAuCl}_{4}$ with sodium citrate by Grabar's method [31]. Briefly, $2.1 \mathrm{~mL}$ of $1 \%$ sodium citrate were added quickly to a boiling $0.03 \% \mathrm{HAuCl}_{4}$ solution $(50 \mathrm{~mL})$, resulting in a change in the color of the solution from pale yellow to deep purplered. The c-NPs were concentrated by centrifugation to an Au concentration equal to $500 \mu \mathrm{g} \mathrm{mL}^{-1}$, as determined by ICP-AES.

\subsubsection{Laser-ablative synthesis of spherical gold nanoparticles (la- NPS)}

Laser-ablative gold nanoparticles (la-NPs) were prepared by pulsed laser ablation using a modified protocol by Kabashin et al. [32] via a Ti/sapphire laser system (Hurricane Spectra Physics Lasers, wavelength $800 \mathrm{~nm}$, repetition rate $1000 \mathrm{~Hz}$, pulse duration $110 \mathrm{fs}$ ). A two-step approach was applied: (i) direct laser ablation of a gold target (Goodfellow, 99.95\%), and (ii) laser fragmentation of the obtained colloidal solution.

During the first step, the gold target was immersed in $18 \mathrm{M} \Omega$ deionized water and irradiated with laser radiation. The liquid volume and its level over the target were $4 \mathrm{~mL}$ and $4 \mathrm{~mm}$, respectively. Laser fluence was controlled by a half-wave plate and was fixed at $150 \mu \mathrm{J} /$ pulse. Radiation was focused on the surface of the target by a $50 \mathrm{~mm}$ lens. In order to avoid ablation from the same area, the gold target was moved constantly. Total ablation time was $20 \mathrm{~min}$. As a result, a colloidal solution of dispersed la-NPs was obtained. In order to narrow their size distribution, a second step of laser fragmentation was used. In this case, the collected solution was irradiated with the same laser radiation focused $10 \mathrm{~mm}$ below the surface level using the same laser fluence. The laser fragmentation step was performed for $1 \mathrm{~h}$ under magnetic stirring. Thus obtained la-NPs were concentrated by centrifugation to an Au

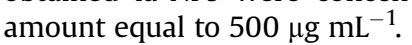

\subsection{Instrumentation}

Infrared spectroscopic studies were performed using a Vertex 70 FTIR spectrometer (Bruker Optics, Ettlingen, Germany) equipped with a BioATRII unit (Bruker Optics, Ettlingen, Germany). The ATR crystal surface interfacing with the sample was made from a circular section of a silicon wafer providing 8-10 internal reflections. IR radiation is coupled into this silicon ATR plate via a secondary zinc selenide (ZnSe) ATR crystal providing a hemispherical shape for efficient coupling of radiation from the FTIR spectrometer into the $\mathrm{Si}$ ATR waveguide. A liquid nitrogen cooled mercury-cadmium-telluride (MCT) detector (Bruker Optics, Ettlingen, Germany) was used for signal recording. Data acquisition and processing was performed using the OPUS 6.5 software package (Bruker Optics, Germany), and the Essential FTIR spectroscopy toolbox (Operant LLC, USA).

Scanning electron microscopy (SEM) images were acquired using a Helios Nanolab 600 scanning electron microscope (FEI Corp., Eindhoven, the Netherlands). The Au concentration was obtained via inductively coupled - atomic emission spectrometry (ICP-AES) using an Ultima 2 system (HORIBA Jobin Yvon, Tokyo, Japan).

\subsection{IR-ATR studies}

For IR-ATR measurements, $20 \mu \mathrm{L}$ of mixtures of aqueous solutions of analyte and AuNPs in equal volumes (1:1) were deposited onto the Si ATR waveguide. Then, spectra were acquired for $1 \mathrm{~min}$ each until total evaporation of the liquid (several hours). IR-ATR spectra were recorded by averaging 100 scans in the spectral range of $4000-500 \mathrm{~cm}^{-1}$ at a spectral resolution of $4 \mathrm{~cm}^{-1}$. For ATR measurements of pure analyte samples, the AuNPs colloid solution was replaced by an equal amount of water, thus keeping the analyte concentration and total sample volume the same. Prior to analyzing each set of samples, a background spectrum was acquired. For better comparability, a baseline correction was performed.

\section{Results and discussion}

\subsection{Characterization of $\mathrm{Au}$ nanostructures}

The morphology of the synthesized $\mathrm{Au}$ nanostructures was investigated via SEM and TEM. As shown in Fig. 1, AuNSts with an outer diameter of approximately $60 \mathrm{~nm}$, which provide approximately 12-15 tips at a length of $15 \mathrm{~nm}$ were obtained.

Bare spherical nanoparticles synthesized by laser ablation (laNPs) had a size distribution ranging from $20 \mathrm{~nm}$ to $40 \mathrm{~nm}$ (Fig. 2a). Despite the absence of any protective ligands, la-NPs colloids remain stable due to electric repulsion effect associated with a strong negative charging of the formed nanoparticles [33]. Citratecapped spherical nanoparticles (c-NPs) with a diameter of approximately $40 \mathrm{~nm}$ (Fig. 2b) were prepared by the reduction of $\mathrm{HAuCl}_{4}$ with sodium citrate, acting both as a reducing agent and as a capping agent following Grabar's method [31].

\subsection{ATR-SEIRA studies at TGA}

FTIR spectra for (a) TGA in water at acidic $\mathrm{pH}$ and (b) the deprotonated form of TGA in a basic solution $(\mathrm{pH} \sim 11.5)$. In the acidic trace (red line), the broad band between 3600 and $2500 \mathrm{~cm}^{-1}$ corresponded to the $\mathrm{O}-\mathrm{H}$ stretch of a carboxylic acid, including the convolution of $\mathrm{C}-\mathrm{H}$ stretching bands with the broad $\mathrm{O}-\mathrm{H}$ feature. The signal at $2550 \mathrm{~cm}^{-1}$ indicates the intact thiol group and the stretch at $1700 \mathrm{~cm}^{-1}$ represents the carbonyl group characteristic of carboxylic acids.

Thioglycolic acid (TGA, HS- $\mathrm{CH}_{2}-\mathrm{COOH}$ ), a.k.a., mercaptoacetic acid has been selected as model analyte for this study. An IR-ATR spectrum of pure TGA is shown in Fig. 3c. The strongest signal around $1709 \mathrm{~cm}^{-1}$ represents the carbonyl band associated with carboxylic acids $(\nu(\mathrm{C}=\mathrm{O}))$ [34]. The $\mathrm{C}-\mathrm{S}$ stretch and the $\mathrm{C}-\mathrm{H}$ bend are located between 685 and $1000 \mathrm{~cm}^{-1}$, respectively. The stretching feature at $2560 \mathrm{~cm}^{-1}$ indicates the intact thiol group, whereas the band at $1145 \mathrm{~cm}^{-1}(\nu(\mathrm{C}-\mathrm{O}))$ and a broad band between 3600 and $2500 \mathrm{~cm}^{-1}$ correspond to the $\mathrm{O}-\mathrm{H}$ stretch of a carboxylic acid, respectively [34,35].

The evaporation kinetics of TGA and mixtures of TGA and Au nanostructures deposited at the surface of the Si waveguide were monitored by in situ ATR-SEIRA. For this purpose, two types of solutions were used: (i) $10 \mu \mathrm{L}$ of AuNSts, c-NPs or la-NPs (initial Au concentration $500 \mu \mathrm{g} \mathrm{mL}^{-1}$ ) and $10 \mu \mathrm{L}$ of TGA (initial TGA concentration $4 \mu \mathrm{g} \mathrm{mL}^{-1}$ ), and (ii) replacing the AuNSt solution with the equivalent amount of water for maintaining a final TGA concentration of $2 \mu \mathrm{g} \mathrm{mL}^{-1}$ and a total volume of $20 \mu \mathrm{L}$, similar to the experiment in the presence of Au nanostructures. In our experiments, we recorded the enhancement of SEIRAS signals for all three 


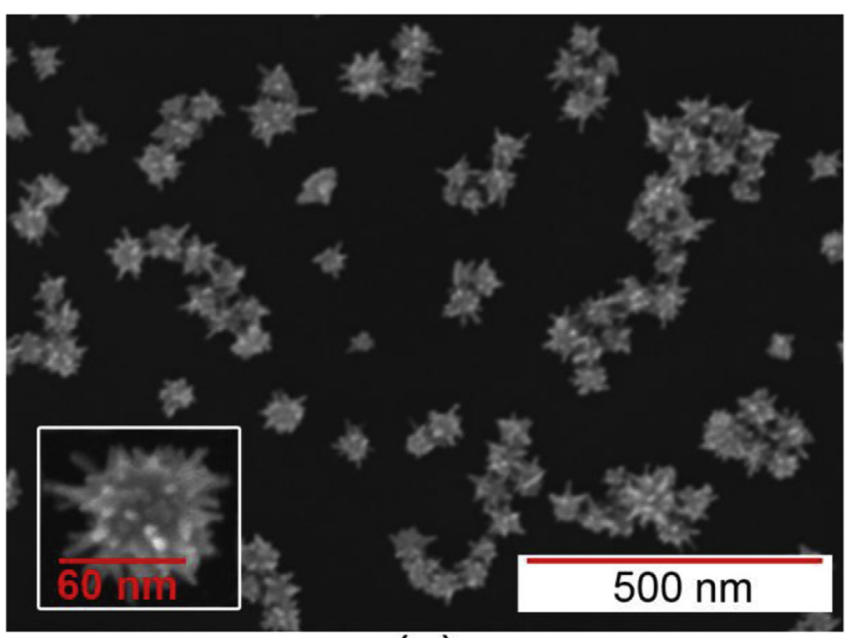

(a)

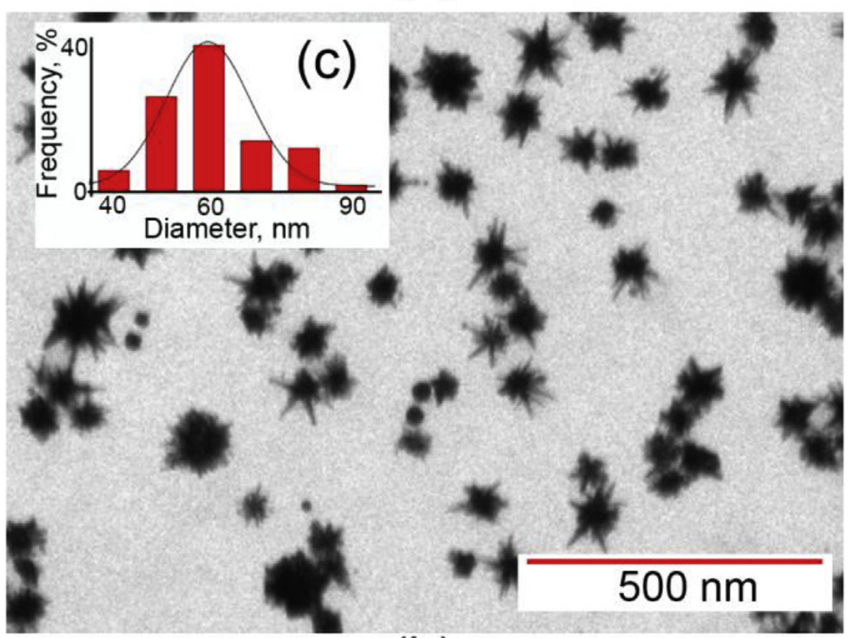

(b)

Fig. 1. SEM (a) and TEM (b) images, and size distribution (c) of gold nanostars (AuNSts).

types of gold nanostructures compared to control samples. Since we monitored the strongest enhancement for gold nanostars, we will mostly present data for these nanostructures, but all conclusions are valid for spherical nanoparticles as well. In the final subsection we summarize and compare data for the investigated nanostructures. Fig. 3 shows exemplary spectra of the TGA solution (a), and of the mixture of TGA and AuNSts (b) vs. time until complete solvent evaporation (red curves) occurs (without baseline correction). A comparison of conventional ATR and ATR-SEIRA spectra of fully evaporated TGA (in black) and TGA + AuNSts (in red) is shown in (d).

Evidently, since air was used as the background spectrum the spectra during the first few minutes are dominated by the water absorption (i.e., $\mathrm{O}-\mathrm{H}$ bending and stretching modes). As water evaporates, a decrease in the water absorption features is observed together with the appearance of the absorption features of TGA, which emerged with enhanced sensitivity if TGA was adsorbed at the AuNSts sedimenting at the Si waveguide surface. The comparison of the enhanced intensity achieved in the IR-ATR spectrum of TGA in the presence of the AuNSts is shown in Fig. 3d.

Minute variances in the TGA spectrum in the wavelength range $1200-1300 \mathrm{~cm}^{-1}$ are explained by the changes in $\mathrm{pH}$ value of the solution [36]. According to the SEM images (Fig. S-1, Supporting

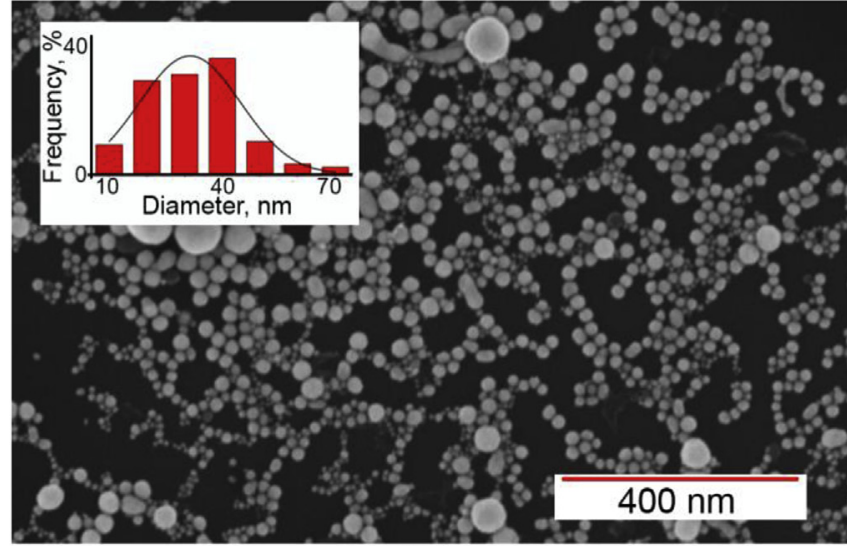

(a)

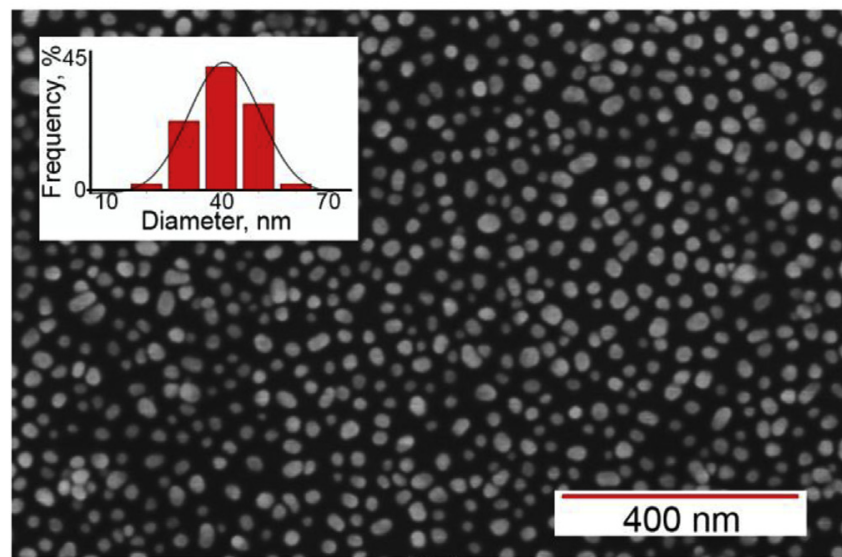

(b)

Fig. 2. (a) SEM image of spherical nanoparticles obtained laser ablation and their size distribution (inset). (b) SEM image of citrate-capped spherical nanoparticles and their size distribution.

Information), AuNSts aggregation during the measurement period was not observed. Detailed information on the detection sensitivity for TGA was obtained by analyzing the strongest band at $1709 \mathrm{~cm}^{-1}$ and the band at $1155 \mathrm{~cm}^{-1}$ to exclude potential overlapping with the water band at $1635 \mathrm{~cm}^{-1}$ caused by the $\mathrm{O}-\mathrm{H}-\mathrm{O}$ scissoring band [37]. The enhancement factor of the SEIRA substrates prepared by simultaneous deposition of TGA and AuNSts, was calculated following [38]:

\section{$\mathrm{EF}=\left(\mathrm{I}_{\text {SEIRAS }} / \mathrm{I}_{\mathrm{ATR}}\right)^{*}\left(\mathrm{~N}_{\mathrm{ATR}} / \mathrm{N}_{\mathrm{SEIRAS}}\right)$,}

where I is the intensity (i.e., band height) of the considered TGA band, and $\mathrm{N}$ represents the total number of analyte molecules deposited on the Si crystal. Taking into account that the TGA concentration was equal in both solutions, an enhancement factor of approximately 10 -fold was calculated considering both TGA bands at $1709 \mathrm{~cm}^{-1}$ and at $1155 \mathrm{~cm}^{-1}$ for a concentration of AuNSts equal to $250 \mu \mathrm{g} \mathrm{mL}^{-1}$ (higher Au concentrations interfere with the quality of the stars). To optimize the experimental parameters and to demonstrate that AuNSts produce the observed SEIRA effect, the relation between the obtained enhancement vs. AuNSts concentration was investigated for two absorption bands, i.e., at $1709 \mathrm{~cm}^{-1}$ (Fig. 4), and at $1155 \mathrm{~cm}^{-1}$ (Fig. S-2, Supporting Information). Each concentration level was analyzed in triplicate with the error bars representing the standard deviation of the mean value.

The analytical performance of the developed SEIRA substrate 


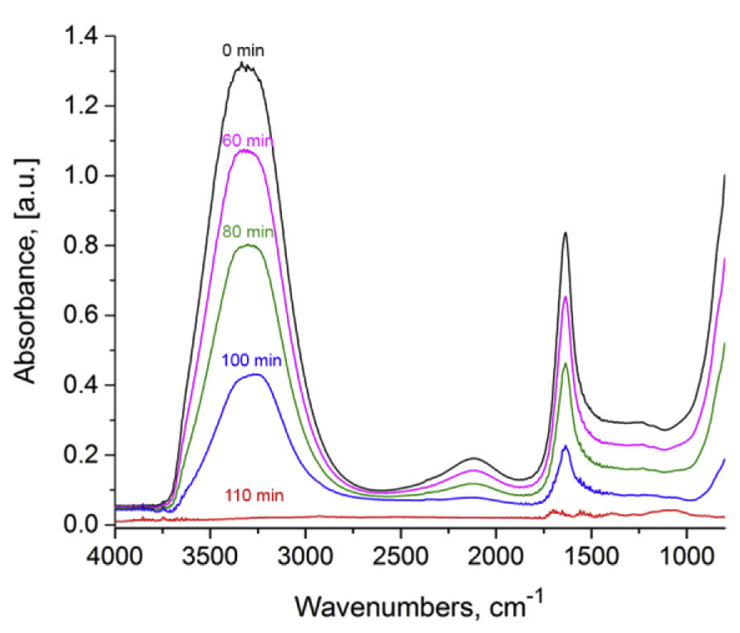

(a)

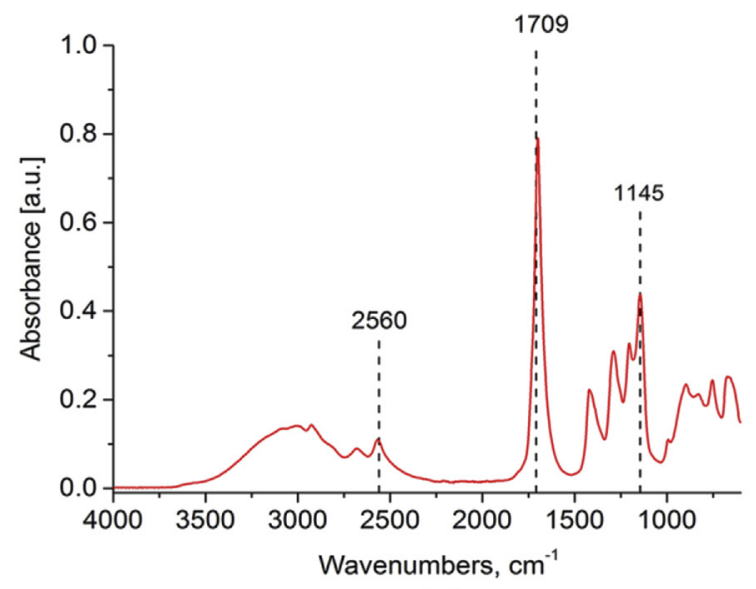

(c)

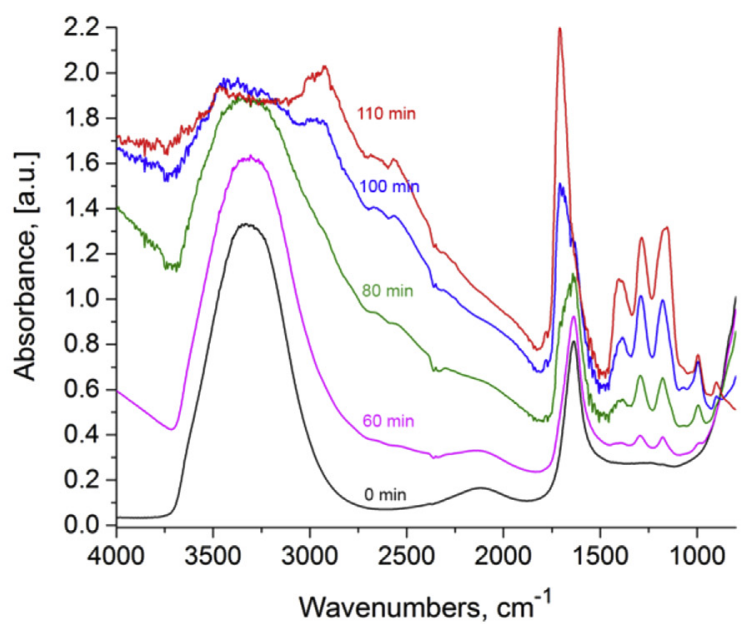

(b)

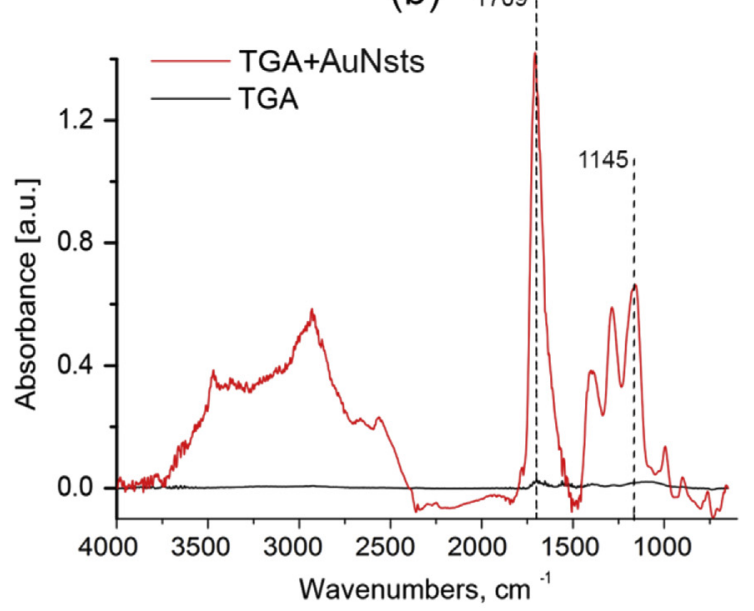

(d)

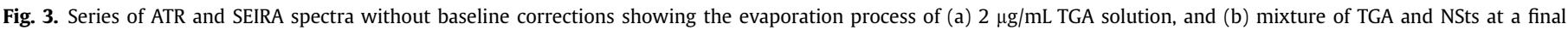

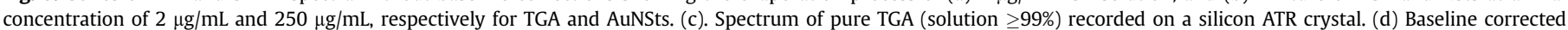

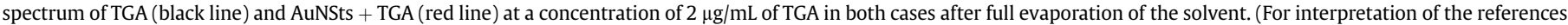
to colour in this figure legend, the reader is referred to the web version of this article.)

was further evaluated by studying TGA and AuNSts + TGA solutions at different concentrations of TGA again depositing $20 \mu \mathrm{L}$ of each solution at the Si waveguide surface after solvent evaporation. A calibration function was established by plotting the height of band at $1709 \mathrm{~cm}^{-1}$ vs. the TGA concentration (Fig. 5).

Each concentration level was analyzed in triplicate with the error bars again representing the standard deviation of the mean value. For comparison, a calibration based on the height of the band at $1155 \mathrm{~cm}^{-1}$ vs. the TGA concentration is represented in Fig. S-3 (Supporting Information).

\subsection{ATR-SEIRA studies at bovine serum albumin}

IR-spectroscopy is an excellent tool for the structural characterization of proteins in various environments. However, the detection of relevant absorptions resulting from protein monolayers is challenging due to the low signal strength [39]. To illustrate the potential of AuNPs in IR biospectroscopy, bovine serum albumin (BSA) was selected as an exemplary analyte. Due to its well-characterized structure and properties, BSA is frequently used as a model protein for fundamental studies of protein-nanoparticle conjugates [40-42].
In situ monitoring of the evaporation kinetics of BSA and BSA mixed with Au nanostructures at the Si crystal surface were performed similar to the TGA studies. The final concentration of BSA was equal to $500 \mu \mathrm{g} \mathrm{mL}^{-1}$ in both BSA and BSA + AuNPs solutions. The AuNSts, c-NPs and la-NPs concentrations were equal to $250 \mu \mathrm{g} \mathrm{mL}{ }^{-1}$. Series of ATR and ATR-SEIRA spectra for AuNSts vs. time during the evaporation process (without baseline correction) are presented in Figs. S-4a and S-4b. A comparison of conventional ATR and ATR-SEIRA spectra of BSA and BSA + AuNSts after solvent evaporation is presented in Fig. 6.

Fig. 6 (black line) shows a native BSA spectrum with the most pronounced bands around $1650 \mathrm{~cm}^{-1}$, which are associated with the amide $\mathrm{I}$ band (i.e., the $\mathrm{C}=\mathrm{O}$ stretching vibrations of the peptide bond), and the band at $1539 \mathrm{~cm}^{-1}$ (i.e., N-H bending vibration/C-N stretching vibration) called the amide II band [43]. The red line in Fig. 6 illustrates spectra of BSA $\left(500 \mu \mathrm{g} \mathrm{mL}^{-1}\right)$ mixed with AuNSts (at a Au mass concentration $250 \mu \mathrm{g} \mathrm{mL} \mathrm{m}^{-1}$ ). A distinct signal enhancement was observed for both, the amide I and amide II band. To avoid any influence of potential water vapor bands located near $1600 \mathrm{~cm}^{-1}$, detailed information on the correlation between the signal enhancement and the AuNSts concentration was obtained by analyzing the amide II band at $1539 \mathrm{~cm}^{-1}$ (Fig. 7). 


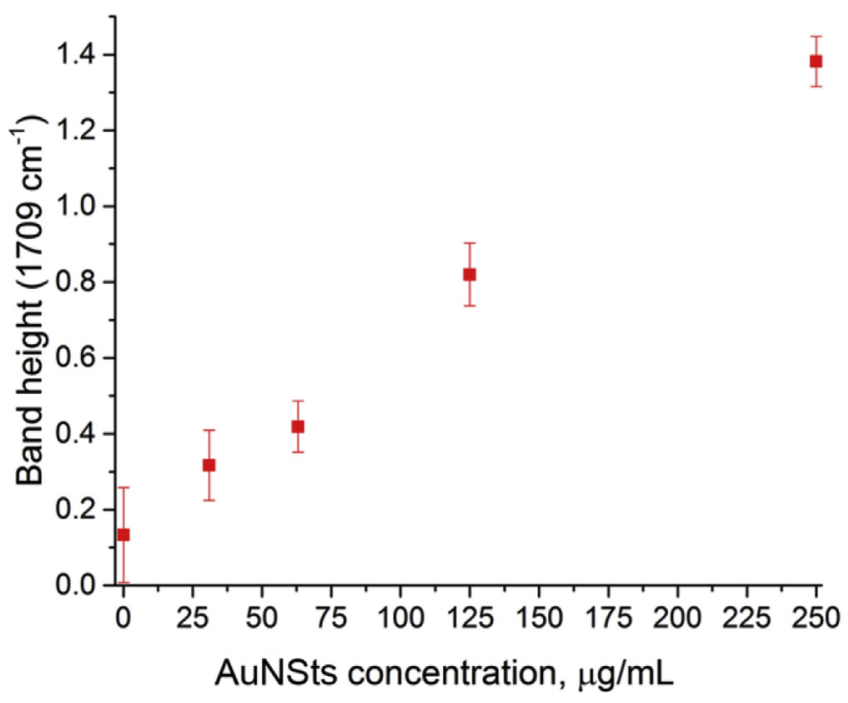

Fig. 4. Height of $1709 \mathrm{~cm}^{-1}$ band of TGA + AuNSts solution versus AuNSts concentration. The maximal concentration of AuNSts $(250 \mu \mathrm{g} / \mathrm{mL})$ caused an absorption enhancement factor of 10 for a TGA concentration of $2 \mu \mathrm{g} / \mathrm{mL}$. Measurements were done after total evaporation of solutions.

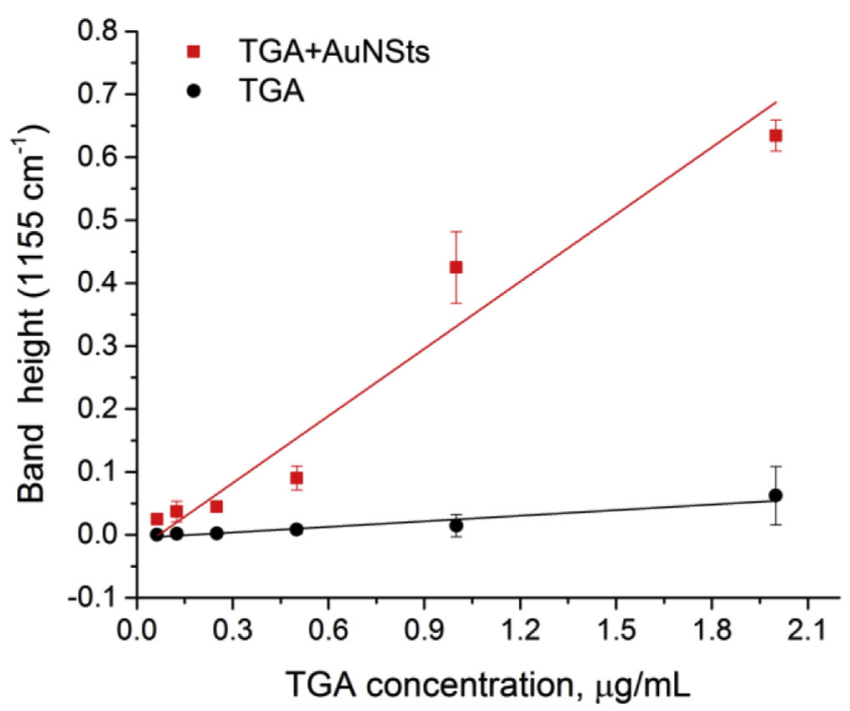

Fig. 5. Calibration graphs at different concentrations of TGA by measuring the height of the band in the ATR and SEIRAS spectra of TGA and TGA + AuNSts at $1709 \mathrm{~cm}^{-1}$. The calibration curves have linear behavior in the chosen concentration range. AuNSts concentration was similar or all samples and equal $250 \mu \mathrm{g} / \mathrm{mL}$.

Analysis of amide I band is presented in Supporting information (Fig. S-5b). As anticipated, the band height increased, as the BSA mass increased from 0 to $250 \mu \mathrm{g} \mathrm{mL}^{-1}$, and gradually saturated for a BSA mass higher then $250 \mu \mathrm{g} \mathrm{mL}^{-1}$. Both curves fit a linear calibration with a goodness of the fit of $\mathrm{r}^{2}=0.993$ for AuNSts + BSA and $\mathrm{r}^{2}=0.996$ for native BSA. However, the saturation process can be fitted with a Langmuir isotherm (Fig. S-6) which represent an adsorption process of the protein within the first monolayer, as also shown in literature [44].

According to SEM images (Fig. S-5a, Supporting Information), AuNSts aggregation during the measurements was not evident. Moreover, absence of a shift of the amide I band position in the spectrum of the AuNSts-BSA mixture in comparison to the native BSA spectrum (Fig. S-5b) suggested that the secondary structure of

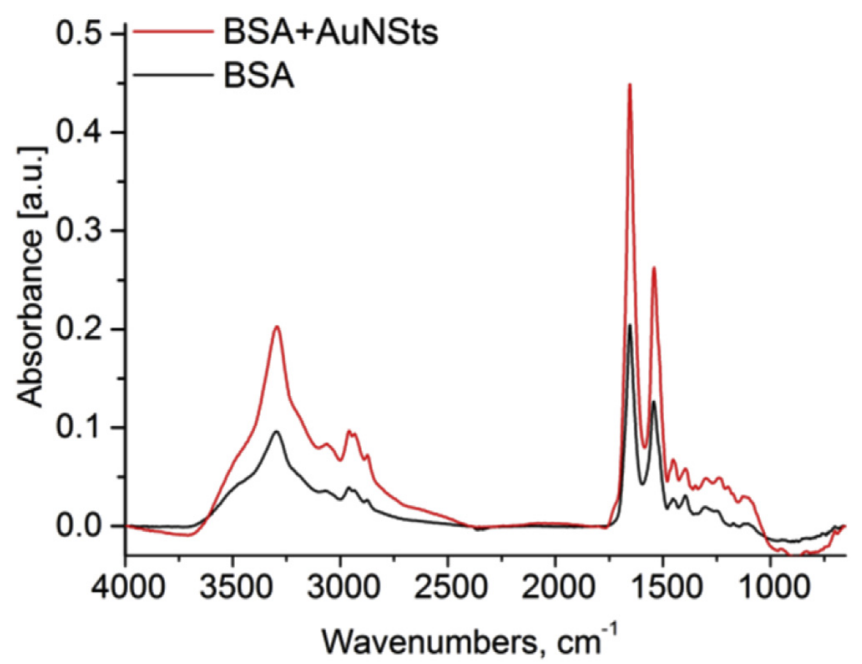

Fig. 6. Spectra of fully evaporated BSA (black line) and AuNSt + BSA (red line) after baseline correction (c). (For interpretation of the references to colour in this figure legend, the reader is referred to the web version of this article.)

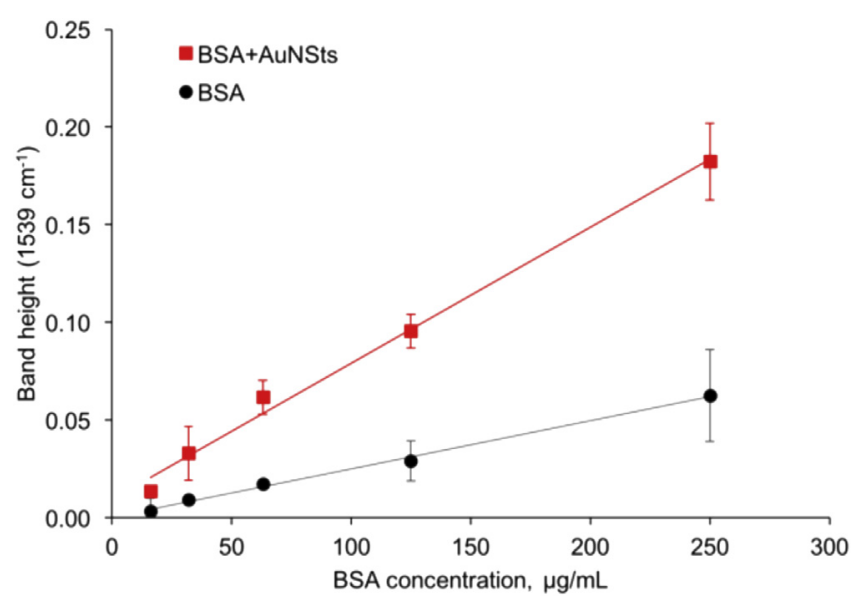

Fig. 7. Calibration at different concentrations of BSA after evaluating conventional ATR and ATR-SEIRA spectra of BSA and BSA + AuNSt at $1539 \mathrm{~cm}^{-1}$. The AuNSt concentration was similar in all samples and equal to $250 \mu \mathrm{g} / \mathrm{mL}$. All measurements were executed after solvent evaporation.

BSA remained unaffected by the presence of AuNSts $[45,46]$ (Table 1).

SEIRA enhancement factors in the same range were demonstrated for vapor-deposited silver (EF between 5 and 150) [47] and platinum (EF around 10) [48] nanofilms, as well as for wet-chemical deposited spherical gold nanoparticles (EF around 5) [49]. The applied EF calculation method was similar to the approach used in

Table 1

Summarizes the analytical figures of merit obtained for the AuNSts enhanced calibration curves of both analytes TGA and BSA, where LOD is the limit of detection; LOQ is the limit of quantification, EF is the enhancement factor and RSD is the relative standard deviation for the highest concentration.

\begin{tabular}{lll}
\hline & TGA $\left(1709 \mathrm{~cm}^{-1}\right)$ & BSA $\left(1539 \mathrm{~cm}^{-1}\right)$ \\
\hline Linear range & $0.063-2 \mu \mathrm{g} \cdot \mathrm{mL}^{-1}$ & $31.25-250 \mu \mathrm{g} \cdot \mathrm{mL}^{-1}$ \\
LOD & $0.3 \mu \mathrm{g} \cdot \mathrm{mL}^{-1}$ & $35 \mu \mathrm{g} \cdot \mathrm{mL}^{-1}$ \\
LOQ & $1 \mu \mathrm{g} \cdot \mathrm{mL}^{-1}$ & $105 \mu \mathrm{g} \cdot \mathrm{mL}^{-1}$ \\
EF & 10 & 2.5 \\
RSD (\%) & 4.42 & 8.4 \\
\hline
\end{tabular}


the present study. Theoretical investigations of nanoshell arrays by finite difference time domain method resulted in electromagnetic SEIRA EF equal to $10^{3}$ [50] or $10^{6}$ [51], while one of the largest SEIRA enhancement factor was experimentally reported as $10^{4}$ [52]. The significant increase in EF factor for AuNSts may be achieved in future by exploiting two contributing effects, i.e., an increase in particle size enhancing the metallic character [53], and an increase in area filling factor; typical EFs for individualized metal nanoparticles are around 25 [54]. Notably, the EF is also strongly depending on the investigated analyte (i.e., TGA and BSA in the present study).

\subsection{Comparison of ATR-SEIRA performance using different types of gold nanostructures}

As we pointed about above, we observed the strongest enhancement of SEIRA signals for gold nanostars, but spherical nanoparticles also provided substantial enhancement. In this section, we summarize enhancement factors for all used nanostructures, namely: anisotropic NSts, bare laser-synthesized gold nanoparticles (la-NPs), and citrate-capped gold nanoparticles (cNPs). In order to achieve similar experimental conditions, the final Au mass for each AuNPs type were brought to the same value. Fig. 8 represents the comparison of the selected band height both for TGA $\left(1709 \mathrm{~cm}^{-1}\right)$ and BSA $\left(1539 \mathrm{~cm}^{-1}\right)$ mixed with three types of GNPs.

One can see that citrate-capped nanoparticles (c-NPs) provided slightly stronger enhancement compared to laser-synthesized nanoparticles (la-NPs), but anisotropic gold nanostars (NSts) outperformed both spherical counterparts. In accordance to SERS experiments, the increased enhancement of the citrate capped nanoparticles in comparison to the laser ablated particles can be assigned to the slightly bigger diameter of $40 \mathrm{~nm}$ of the citrate capped ones versus the $30 \mathrm{~nm}$ of the laser ablated ones [55]. Furthermore, citrate capped nanoparticles innately might hamper analyte adsorption due to the citrate shell, an exchange can be anticipated within the early stages after solution mixing and therefore can be neglected at the experiment time scales [56]. Indeed, SEIRA signals from TGA were about two times stronger for NSts compared to spherical nanoparticles (Fig. 8a). Although the difference in SEIRA signals for BSA was not so significant, it still reached $20-25 \%$ (Fig. 8b). Specific sulfur-gold interaction between TGA and gold nanoparticles is expected. Although the same amount of Au was applied for TGA and BSA, the enhanced band height may partially ascribe to the increased total surface area of nanoparticles. This may account for the less pronounced enhancement factor observed for BSA which has a large molecular volume and no specific interaction with Au. We believe that such a difference in enhancement factors is due to a much larger electromagnetic field strength at sharp tips on the surface of nanostars. Thus, our experiments demonstrated that NSts the most applicable enhancer agents for Surface enhanced vibrational spectroscopy (SEVS).

\section{Conclusions}

Various plasmonic nanostructures, including anisotropic nanostars (NSts) and spherical nanoparticles, were applied for the detection of exemplary analytes in ATR-SEIRA spectroscopy providing enhanced spectral signatures in the infrared regime. This approach enables the detection of molecules close to or at the surface of nanoparticles deposited at Si ATR waveguide surfaces improving the sensitivity of detection by up to 10 -fold. In comparison to spherical gold nanoparticles, AuNSts confirmed superior enhancement due to their anisotropic surface structure. The obtained results demonstrate the utility of Au nanostructures in ATR-

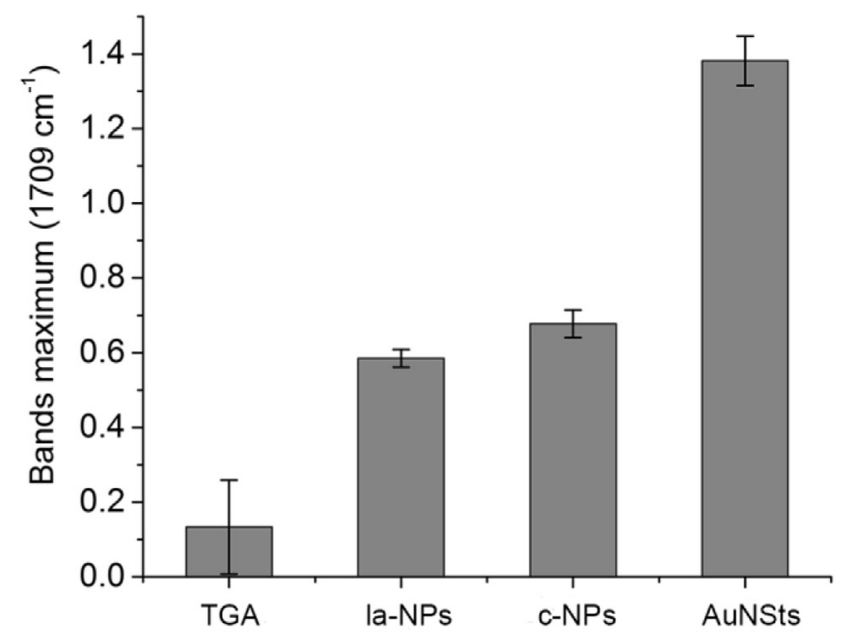

(a)

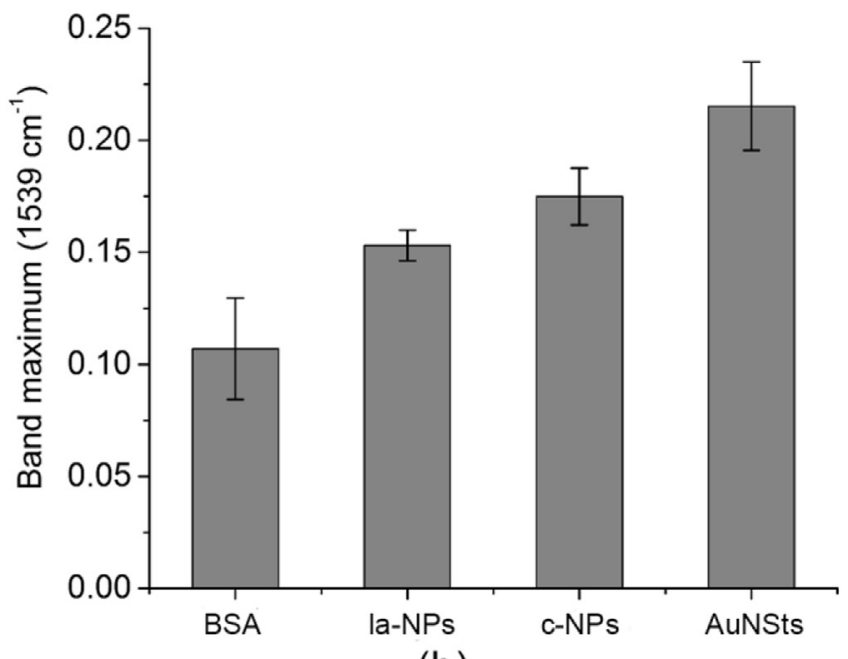

(b)

Fig. 8. (a) Band height at $1709 \mathrm{~cm}^{-1}$ of evaporated TGA solution and TGA mixed with laser-synthesized nanoparticles (la-NPs), citrate-capped nanoparticles (c-NPs) and AuNSts. (b) Band height at $1539 \mathrm{~cm}^{-1}$ of evaporated BSA solution and BSA mixed with ultrapure la-NPs, c-NPs and AuNSts. Concentration of each type of GNPs was kept as $250 \mu \mathrm{g} / \mathrm{mL}$. All measurements were executed after solvent evaporation.

SEIRA applications, which is considered of relevance in IR chem/bio sensing schemes as well es IR biospectroscopy, e.g., of proteins and biomarkers. It is anticipated that these findings will aid in enhancing the sensitivity of label-free IR assay technologies via deliberately controlled SEIRA-based signal amplification. This is of particular importance, if extremely low biomarker concentrations have to be detected, as encountered, e.g., in neurodegenerative diseases such as Parkinson or Alzheimer disease. Given the obtained improvements in signal intensity, IR spectroscopy and chem/ bio sensing concepts may emerge as a viable alternative vs. more time- and resource-consuming screening technologies currently implemented in conventional medical diagnostic scenarios. Moreover, the number of potential interferants in the MIR requires substantial studies on the competitive behavior of overlapping spectral features by matrix molecules in close vicinity of the SEIRA substrate. Hence, subsequent studies will follow up in detail on these aspects, in order to prove the applicability of the presented SEIRA substrate in real-world scenarios. 


\section{Conflict of interest}

The authors declare no competing financial interest.

\section{Acknowledgments}

O. Bibikova acknowledges the support from Infotech Oulu Graduate School and International Graduate School in Molecular Medicine Ulm (IGradU), the program "Tissue Homeostasis: Development, Aging and Regeneration, TissueHome”. A.I. López-Lorente thanks the Alexander von Humboldt Foundation for the award of a Postdoctoral Fellowship at the Institute of Analytical and Bioanalytical Chemistry (University of Ulm, Germany). Funding by the Horizon 2020 Framework Program of the European Union within the MSCA RISE Project TROPSENSE [grant number 645758] is greatly acknowledged. A. Popov acknowledges Academy of Finland for partial financial support [grant numbers 260321, 290596]. This work was also partially supported by Government of Russian Federation [grant numbers 074-U01]. Finally, Yu. Ryabchikov and A. V. Kabashin acknowledge a support from LASERNANOCANCER [grant number PC201420]; GRAVITY projects of the ITMO "Plan Cancer 2014-2019" INSERM program; AMIDEX project [grant number ANR-11-IDEX-0001-02] funded by the French "Investissements d'Avenir" French Government program.

\section{Appendix A. Supplementary data}

Supplementary data related to this article can be found at http:// dx.doi.org/10.1016/j.aca.2017.07.045.

\section{References}

[1] S. Eustis, M.A. El-Sayed, Why gold nanoparticles are more precious than pretty gold: noble metal surface plasmon resonance and its enhancement of the radiative and nonradiative properties of nanocrystals of different shapes, Chem. Soc. Rev. 35 (2006) 209-217.

[2] A. Harstein, J.R. Kirtley, J.C. Tsang, Enhancement of the infrared absorption from molecular monolayers with thin metal overlayers, Phys. Rev. Lett. 45 (1980) 201-204.

[3] R. Adato, H. Altug, In-situ ultra-sensitive infrared absorption spectroscopy of biomolecule interactions in real time with plasmonic nanoantennas, Nat. Commun. 4 (2013) 2154.

[4] N.J. Harrick, InternaI Reflection Spectroscopy, Interscience, New York, 1967.

[5] W.N. Hansen, in: P. Delahay, C.W. Tobias (Eds.), Advanced in Electrochemistry and Electrochical Engineering, John Wiley, New York, 1973.

[6] R. Kellner, B. Mizaikoff, M. Jakusch, H.D. Wanzenbock, N. Weissenbacher, Surface-enhanced vibrational spectroscopy: a new tool in chemical IR sensing? Appl. Spectrosc. 51 (1997) 495-503.

[7] X. Jiang, E. Zaitseva, M. Schmidt, F. Siebert, M. Engelhard, R. Schlesinger, K. Ataka, R. Vogel, J. Heberle, Resolving voltage-dependent structural changes of a membrane photoreceptor by surface-enhanced IR difference spectroscopy, Proc. Natl. Acad. Sci. U.S.A. 105 (2008) 12113.

[8] B. Jin, W.J. Bao, Z.Q. Wu, X.H. Xia, In situ monitoring of protein adsorption on a nanoparticulated gold film by attenuatedtotal reflection surface-enhanced infrared absorption spectroscopy, Langmuir 28 (2012) 9460-9465.

[9] K. Ataka, J. Heberle, Functional vibrational spectroscopy of a cytochrome c monolayer: SEIDAS probes the interaction with different surface-modified electrodes, J. Am. Chem. Soc. 126 (2004) 9445-9457.

[10] Á.I. López-Lorente, M. Sieger, M. Valcárcel, B. Mizaikoff, Infrared attenuated total reflection spectroscopy for the characterization of gold nanoparticles in solution, Anal. Chem. 86 (2014) 783-789.

[11] A.I. López-Lorente, B.M. Simonet, M. Valcárcel, S. Eppler, S. Schindl, C. Kranz, B. Mizaikoff, Characterization of stainless steel assisted bare gold nanoparticles and their analytical potential, Talanta 118 (2014) 321-327.

[12] R. Adato, A.A. Yanik, J.J. Amsden, D.L. Kaplan, F.G. Omenetto, M.K. Hong, S. Erramilli, H. Altug, Ultra-sensitive vibrational spectroscopy of protein monolayers with plasmonic nanoantenna arrays, PNAS 106 (2009) 19227-19232.

[13] F. Le, D.W. Brandl, Y.A. Urzhumov, H. Wang, J. Kundu, N.J. Halas, J. Aizpurua, P. Nordlander, Metallic nanoparticle arrays: a common substrate for both surface-enhanced Raman scattering and surface-enhanced infrared absorption, ACS Nano 2 (2008) 707-718.

[14] J.M. Delgado, J.M. Orts, J.M. Perez, A.J. Rodes, Sputtered thin-film gold electrodes for in situ ATR-SEIRAS and SERS studies, Electroanal. Chem. 617 (2008) 130-140.
[15] J.M. Hoffmann, H. Janssen, D.N. Chigrin, T. Taubner, Enhanced infrared spectroscopy using small-gap antennas prepared with two-stepevaporation nanosphere lithography, Opt. Express 22 (2014) 14425-14432.

[16] H.D. Wanzenböck, B. Edl-Mizaikoff, G. Friedbacher, M. Grasserbauer R. Kellner, M. Arntzen, T. Luyven, W. Theiss, P. Grosse, Surface-enhanced infrared absorption spectroscopy (SEIRA) using multireflection ATR-elements, Mikrochim. Acta 14 (1997) 665-667.

[17] J.A. Seelenbinder, C.W. Brown, P. Pivarnik, A.G. Rand, Colloidal gold filtrates as metal substrates for surface-enhanced infrared absorption spectroscopy, Anal Chem. 71 (1999) 1963-1966.

[18] F. Le, D.W. Brandl, Y.A. Urzhumov, H. Wang, J. Kundu, N.J. Halas, J. Aizpurua P. Nordlander, Metallic nanoparticle arrays: a common substrate for both surface-enhanced Raman scattering and surface-enhanced infrared absorption, ACS Nano 2 (2008) 707-718.

[19] B. Nikoobakht, M.A. El-Sayed, Preparation and growth mechanism of gold nanorods (nrs) using seed-mediated growth method, Chem. Mater 15 (2003) 1957-1962.

[20] S.J. Oldenburg, S.L. Westcott, R.D. Averitt, N.J. Halas, Surface enhanced Raman scattering in the near infrared using metal nanoshell substrates, J. Chem. Phys. 111 (1999) 4729.

[21] S.E. Skrabalak, J. Chen, Y. Sun, X. Lu, L. Au, C.M. Cobley, Y. Xia, Gold nanocages: synthesis, properties, and applications, Acc. Chem. Res. 41 (2008) 1587-1595.

[22] H. Yuan, C.G. Khoury, H. Hwang, Ch M. Wilson, G.A. Grant, T. Vo-Dinh, Gold nanostars: surfactant-free synthesis, 3D modelling, and two-photon photoluminescence imaging, Nanotechnology 23 (2012) 075102.

[23] E. Prodan, C. Radloff, N.J. Halas, P. Nordlander, A hybridization model for the plasmon response of complex nanostructures, Science 302 (2003) 419-422.

[24] G. Chirico, M. Borzenkov, P. Pallavicini, Gold Nanostars Synthesis, Properties and Biomedical Application, Springer Briefs in Materials, New York, 2015.

[25] A. Kedia, P.S. Kumar, Precursor-driven nucleation and growth kinetics of gold nanostars, J. Phys. Chem. C 116 (2012) 1679-1686.

[26] P.M. Favi, M. Gao, L.J.S. Arango, S.P. Ospina, M. Morales, J.J. Pavon, T.J. Webster, Shape and surface effects on the cytotoxicity of nanoparticles: gold nanospheres versus gold nanostars, J. Biomed. Mater. Res. A 103 (2015) 3449-3462.

[27] A.I. Busev, Analytical Chemistry of Molybdenum, Daniel Davy and Co., Inc., New York, N. Y., 1964, p. p 52.

[28] A.G. Young, D.P. Green, A.J. McQuillan, Infrared spectroscopic studies of monothiol ligand adsorption on CdS nanocrystal films in aqueous solutions, Langmuir 22 (2006) 11106-11112.

[29] R. Lu, W.-W. Li, A. Katzir, Y. Raichlin, H.-O Yu, B. Mizaikoff, Probing the secondary structure of bovine serum albumin during heat -induced denaturation using mid-infrared fiberoptic sensors, Analyst 140 (2015) 765.

[30] O. Bibikova, A. Popov, A. Bykov, A. Fales, H. Yuan, I. Skovorodkin, M. Kinnunen, S. Vainio, T. Vo-Dinh, V. Tuchin, I. Meglinski, Plasmon-resonant gold nanostars with variable size as contrast agents for imaging applications, IEEE J. Sel. Top. Quantum Electron 22 (2016) 1-8.

[31] K.C. Grabar, R.G. Freeman, M.B. Hommer, M. Natan, Preparation and characterization of Au colloid monolayers, J. Anal. Chem. 67 (1995) 735-743.

[32] A.V. Kabashin, M. Meunier, Femtosecond laser ablation in aqueous solutions: a novel method to synthesize non-toxic metal colloids with controllable size, J. Phys. Conf. Ser. 59 (2007) 354.

[33] K. Maximova, A. Aristov, M. Sentis, A.V. Kabashin, Size-controllable synthesis of bare gold nanoparticles by femtosecond laser fragmentation in water, Nanotechnology 26 (2015) 065601.

[34] J.L. Castro, M.R. Lopez-Ramirez, S.P. Centeno, J.C. Otero, Adsorption of mercaptoacetic acid on a colloidal silver surface as investigated by Raman spectroscopy, Biopolymers 74 (2004) 141-145.

[35] A.R. Attar, D.E. Blumling Jr., K.L. Knappenberger, Photodissociation of thioglycolic acid studied by femtosecond time-resolved transient absorption spectroscopy, J. Chem. Phys. 134 (2011) 024514.

[36] A. Krolikowska, A. Kudelski, A. Michota, J. Bukowska, SERS studies on the structure of thioglycolic acid monolayers on silver and gold, Surf. Sci. 32 (2003) 532-535.

[37] B.L. Mojet, S.D. Ebbesenz, L. Lefferts, Light at the interface: the potential of attenuated total reflection infrared spectroscopy for understanding heterogeneous catalysis in water, Chem. Soc. Rev. 39 (2010) 4643-4655.

[38] W.W. Yu, I.M. White, A simple filter-based approach to surface enhanced Raman spectroscopy for trace chemical detection, Analyst 137 (2012) 1168-1173.

[39] Á.I. López-Lorente, B. Mizaikoff, Mid-infrared spectroscopy for protein analysis: potential and challenges, Anal. Bioanal. Chem. (2016) 2875-2889.

[40] K. Kaibara, T. Okazaki, H.B. Bohidar, P.L. Dubin, pH-induced coacervation in complexes of bovine serum albumin and cationic polyelectrolytes, Biomacromolecules 1 (2000) 100-107.

[41] D.C. Carter, X.J. Ho, Structure of serum albumin, Adv. Protein Chem. 45 (1994) 153-203.

[42] A. Papadopoulou, R.J. Green, R.A. Frazier, Interaction of flavonoids with bovine serum albumin: a fluorescence quenching study, J. Agric. Food Chem. 53 (2005) 158-163.

[43] Á.I. López-Lorente, B. Mizaikoff, Mid-infrared spectroscopy for protein analysis: potential and challenges, Anal. Chem. 408 (2016) 2875-2889.

[44] A.I. López-Lorente, P. Wang, B. Mizaikoff, Towards label-free mid-infrared protein assays: in-situ formation of bare gold nanoparticles for surface enhanced infrared absorption spectroscopy of bovine serum albumin, 
Microchim. Acta 184 (2017) 453-462.

[45] L. Shang, Y. Wang, J. Jiang, S. Dong, pH-dependent protein conformationa changes in albumin: gold nanoparticle bioconjugates: a spectroscopic study, Langmuir 23 (2007) 2714-2721.

[46] Y. Yue, H.-W. Li, T.-Y. Liu, Y. Wu, Vib. Spectrosc. 74 (2014) 137-141.

[47] Michelle M. Killian, E. Villa-Aleman, Z. Sun, S. Crittenden, Ch L. Leverette, Dependence of surface-enhanced infrared absorption (SEIRA) enhancement and spectral quality on the choice of underlying substrate: a closer look at silver (Ag) films prepared by physical vapor deposition (PVD), Appl. Spectrosc 65 (2011) 272-283.

[48] A.E. Bjerke, P.R. Griffiths, Surface-enhanced infrared absorption spectroscopy of p-nitrothiophenol on vapor-deposited platinum films, Appl. Spectrosc. 56 (2002) 1275-1280.

[49] N. Kumar, S. Thomas, R.B. Tokas, R.J. Kshirsagar, SEIRA studies of uracil adsorbed on wet-chemically prepared gold nanoparticles film on glass substrate - effect of morphology of film, Spectrochim. Acta Mol. Biomol. Spectrosc. 129 (2014) 359-364.

[50] D.W. Brandl, Y.A. Urzhumov, H. Wang, J. Kundu, N.J. Halas, J. Aizpurua, Metallic nanoparticle arrays: a common substrate for both surface-enhance raman scattering and surface-enhanced infrared absorption, ACS Nano 2
(2008) 707-718

[51] H. Wang, J.- Kundu, N.-J. Halas, Plasmonic nanoshell arrays combine surfaceenhanced vibrational spectroscopies on a single substrate, Angew. Chem. Int. Ed. 46 (2007) 9040-9044.

[52] J. Kundu, F. Le, P. Nordlander, N.J. Halas, Surface enhanced infrared absorption (SEIRA) spectroscopy on nanoshell aggregate substrates, Chem. Phys. Let. 452 (2008) 115-119.

[53] A. Pucci, IR spectroscopy of adsorbates on ultrathin metal films, Phys. Status Solidi B 242 (2005) 2704-2713.

[54] D. Enders, A. Pucci, Surface enhanced infrared absorption of octadecanethiol on wet-chemically prepared Au nanoparticle films, App. Phys. Lett. 88 (2006) 184104.

[55] K. Sugawa, T. Akiyama, Y. Tanoue, T. Harumoto, S. Yanagida, A. Yasumori, S. Tomita, J. Otsuki, Particle size dependence of the surface-enhanced Raman scattering properties of densely arranged two-dimensional assemblies of Au(core)-Ag(shell) nanospheres, Phys. Chem. 17 (2015) 21182-21189.

[56] K. Siriwardana, M. Gadogbe, S.M. Ansar, E.S. Vasquez, W.E. Collier, S. Zou, K.B. Walters, D. Zhang, Ligand adsorption and exchange on pegylated gold nanoparticles, J. Phys. Chem. C 118 (2014) 11111-11119. 